Compurter schence

\title{
Vit:
}

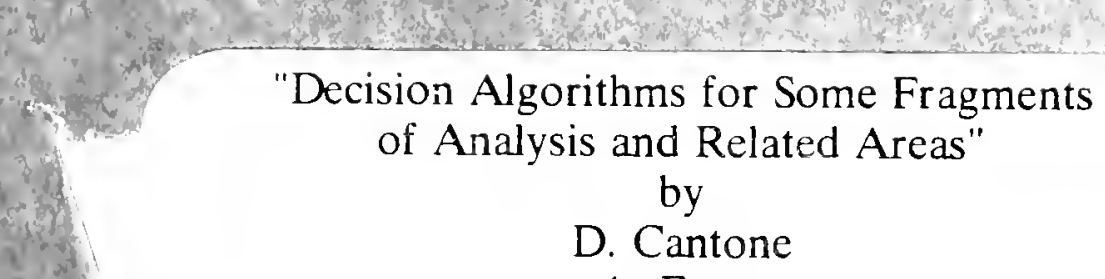

A. Ferro

E. Omodeo

J. T. Schwartz†

Technical Report \#217

May, 1986

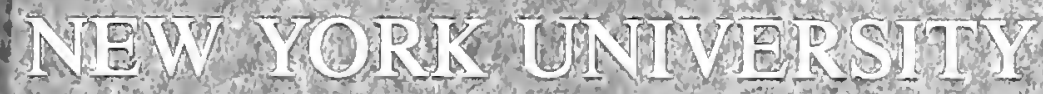

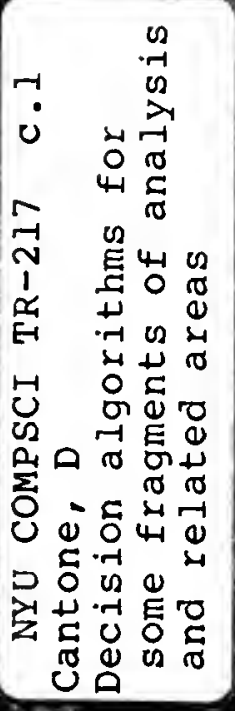




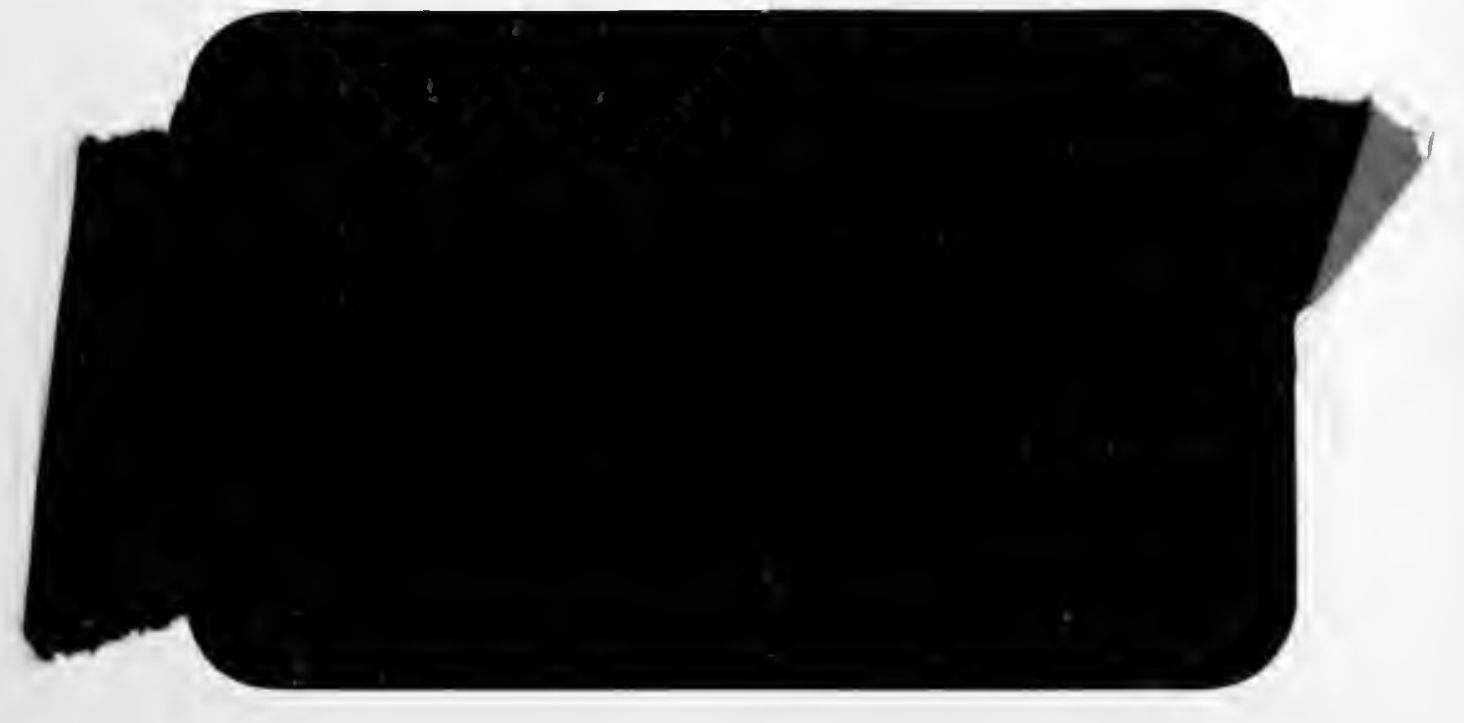




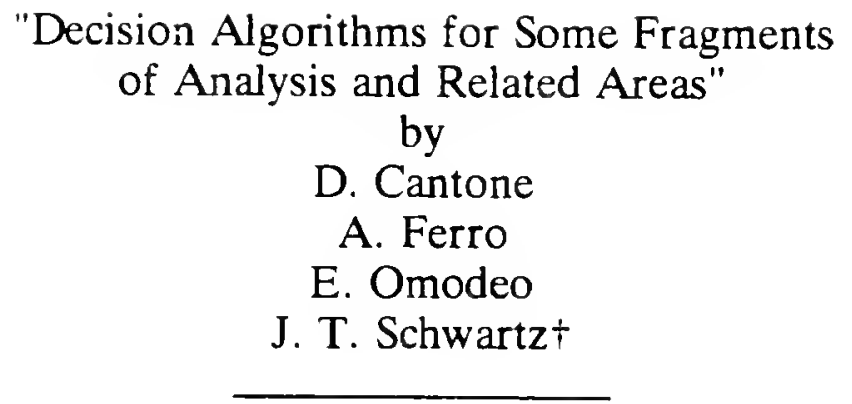

Technical Report \#217

May, 1986

$\dagger$ This research is supported in part by the National Science Foundation under Grant No. MCS-8300631. 


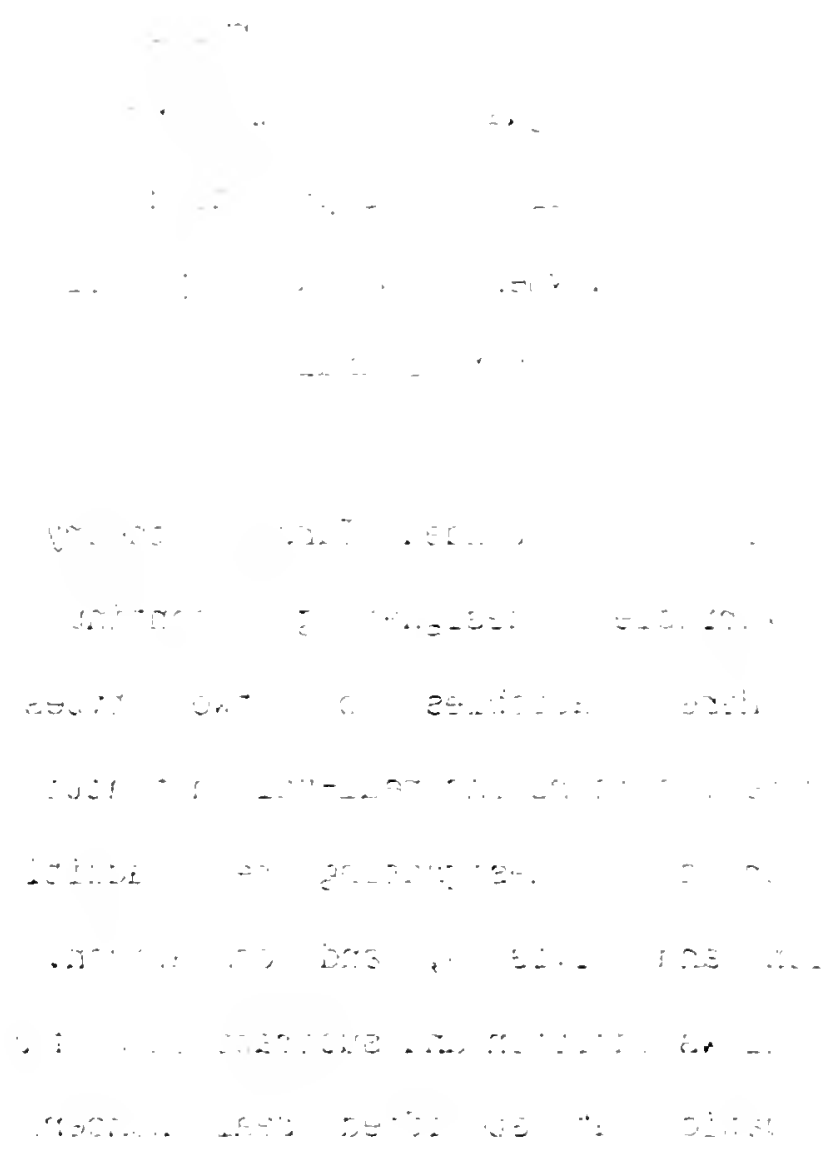


D. Cantone, A. Ferro, E. Omodeo and J. T. Schwartz

1. Introduction

This paper gives (relatively straightforward) decision algorithms for some fragmentary sublanguages of elementary analysis and related fields. It is felt that the algorithms presented could form a useful part of the 'initial endowment' of a computerized proof verifier intended to facilitate proofs in real analysis. The specific languages treated are

(i) The (unquantified) 'extended Tarski' theory of reals with additional variables designating continuous real-valued functions. Here variables of two types, respectively representing real numbers and real-valued functions, exist, along with operation signs designating real addition, subtraction, multiplication and division, and comparison. Moreover, the theory also allows addition and subtraction of functions, as well as their evaluation at specified real numbers, and moreover allows positivity, monotonicity and convexity of functions to be asserted.

(ii) A multi-dimensional extension of theory (i).

(iii) More abstract theories of totally and partially ordered sets and monotone functions on them.

In all cases, decidability is shown using a standard reduction process, which proceeds by applying a series of transformations to an

This research is supported in part by the National Science Founaation under Grant No. MCS -8300631 . 





initial formula in such a way as to preserve satisfiability but which is such that the resulting formula belongs to a theory for which a decidability algorithm is already known. For example, for language (i) we reduce formulae to formulae in the elementary theory of reals, which is known to be decidable.

2. The normalization process

Let $\mathrm{T}$ be an unquantified theory with equality =, variables $\mathrm{x}, \mathrm{y}, \ldots$, function symbols $\mathrm{f}_{1}, \mathrm{f}_{2}, \ldots$, and predicate symbols $\mathrm{P}_{1}, \mathrm{P}_{2}, \ldots$.

Definition 2.1. A formula $\phi$ of $T$ is in normal form if:

(i) all terms occurring in $\phi$ have the simple form $f\left(x_{1}, \ldots, x_{n}\right)$ or just $x$, where $x, x_{1}, \ldots, x_{n}$ are variables and $f$ is a function symbol;

(ii) all atoms are either of the form $x=t$, where $x$ is a variable and $t$ a term, or of the form $P\left(x_{1}, \ldots, x_{n}\right)$, where $x_{1}, \ldots, x_{n}$ are variables of appropriate types and $P$ is a predicate symbol which applies to variables of these types.

In dealing with the decidability problems of any theory, it is advantageous to be able to concentrate on formulae in normal form. To transform any formula into a normal form formula such that the initial and resulting formula are equisatisfiable, we use the following easy result.

Lemma 2.2. There is an effective procedure to transform any formula $\phi$ in $T$ to a formula $\psi$ in normal form such that $\phi$ and $\psi$ are equisatisfiable.

Proof: Consider the following

Normalization Algorithm 


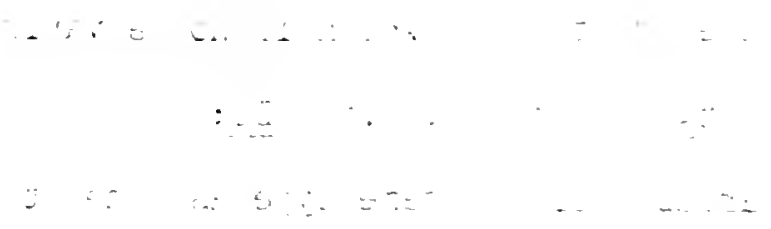


WHILE there is an atom of the form $t_{S}=t$, or there is an atom of the

form $P_{1}\left(t_{1}, \ldots, t_{s-1}, t_{s}, t_{s+1}, \ldots t_{n}\right)$ where $t_{1}, \ldots, t_{n}, t$ are terms and $t_{S}$, $t$ are not variables $\underline{D O}$

Let $x_{t_{S}}$ be a new variable of the same type as $t_{S}$ and not occurring in $\phi$. Ada to $\phi$ the formula $x_{t_{S}}=t_{s}$ and substitute the variable $x_{t_{S}}$ wherever the term $t_{S}$ occurs in $\phi$.

\section{END WHILE;}

[Comment: At this point part (ii) of Definition 2.1 holds. To satisfy also part (i) we perform the following loop.]

WHILE there is a proper subterm $t_{S}$ which is not a variable occurring in a term $f\left(t_{1}, \ldots, t_{s-1}, t_{s}, t_{s+1}, \ldots, t_{n}\right)$ DO:

Let $x_{t_{s}}$ be a variable of the same type as the term $t_{s}$ and not occurring in $\phi$.

Add to $\phi$ the formula $x_{t_{s}}=t_{s}$ and substitute the variable $x_{t_{s}}$ wherever a term $t_{S}$ occurs.

END WHILE;

It is clear that each step of the above algorithm preserves satisfiability. Therefore the final formula $\psi$ produced by the algorithm satisfies the assertion of the lemma.

Definition 2.3. A normal form formula $\phi$ of $t$ is a standard normal form formula if it is a conjunction of literals of the types

$\Leftrightarrow \quad x_{1}=x_{2}, \quad x_{1}=f\left(y_{1}, \ldots, y_{n}\right)$

$(\neq) \quad x_{1} \neq x_{2}$

(P) $\quad P\left(x_{1}, \ldots, x_{n}\right)$

$(\mid P) \quad-\mid P\left(x_{1}, \ldots, x_{n}\right)$,

where $x_{1}, \ldots, x_{n}, y_{1}, \ldots, y_{n}$ are variables. 


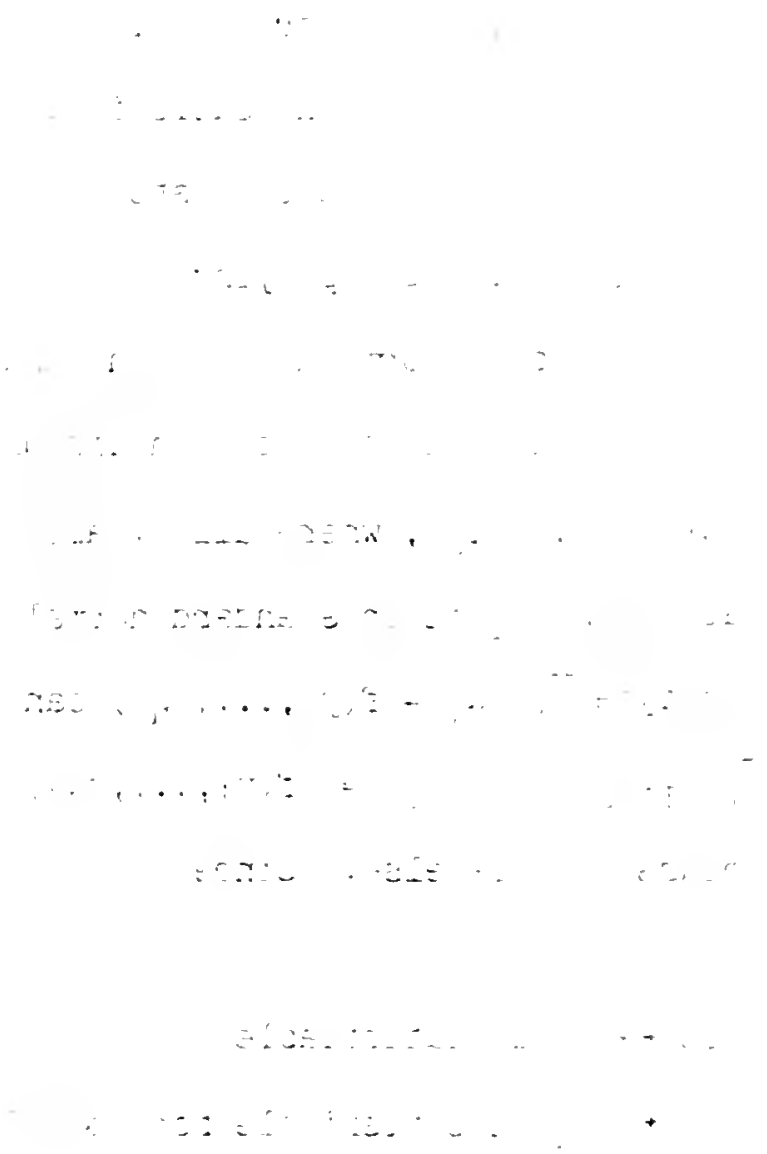


Let $S$ be the class of all formulae of $T$ in standard normal form. Then we have the following

Lemma 2.4. $T$ is decidable if and only if $S$ is decidable.

Proof: Clearly any algorithm for testing satisfiability of formulae in $T$ is an algorithm for testing satisfiability of formulae in S. Conversely let $\underline{A}$ be an algorithm which tests satisfiability in $S$, and let $\phi$ be any formula in T. By applying the normalization process to $\phi$, we get a normal form formula $\psi$ such that $\phi$ and $\psi$ are equisatisfiable. At this point we can put $\psi$ in disjunctive normal form obtaining a formula $\psi_{1} \vee \ldots \vee \psi_{n}$, where all $\psi_{i}$ are conjunctions, and we may assume that each $\psi_{i}$ is in standard normal form, because any literals in it of the type $\mid\left(x_{1}=f\left(y_{1}, \ldots, y_{n}\right)\right)$ can be replaced by the conjunction $-\mid\left(x_{1}=x_{2}\right) \&\left(x_{2}=f\left(y_{1}, \ldots, y_{n}\right)\right)$ by introducing a variable $x_{2}$ which occurs nowhere else. Since

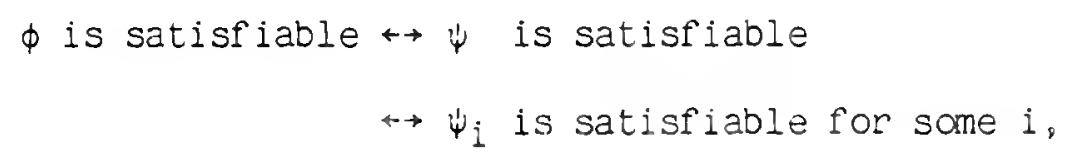

and since all transformations used to build conjunctions $\psi_{1}, \ldots, \psi_{n}$ are effective, our lemma follows. Q.E.D.

In the following sections we will use these elementary concepts and results freely, without specific reference. 


$$
\begin{aligned}
& \text {..T }-\cdots,=
\end{aligned}
$$

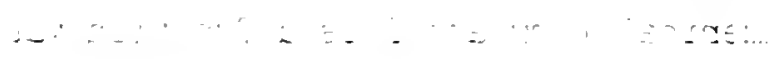

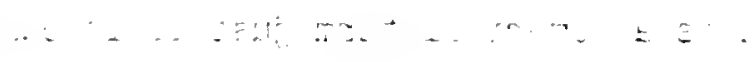

$$
\begin{aligned}
& \therefore \cdots, \because \ldots ; \quad \because \ldots
\end{aligned}
$$

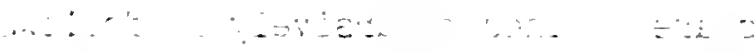




3. 'Extended Tarski' theory of reals with continuous functions.

In the extension of Tarski theory of reals considered in this section we allow two kinds of variables, namely real variables, $\mathrm{x}, \mathrm{y}, \ldots$, and function variables $\mathrm{f}, \mathrm{g}, \ldots$. Constants 0,1 , both numerical and functional are also allowed. We distinguish two kinds of terms, namely numerical terms and functional terms, as follows:

Definition 3.1. Numerical terms are defined recursively by:

(i) Any real variable $\mathrm{x}, \mathrm{y}, \ldots$ or constant 0,1 is a numerical term.

(ii) If $t_{1}, t_{2}$ are numerical terms, then $\left(t_{1}+t_{2}\right),\left(t_{1}-t_{2}\right),\left(t_{1} \cdot t_{2}\right)$, $\left(t_{1} / t_{2}\right)$ are numerical terms.

(iii) If $t$ is a numerical term and $f$ is a function variable, then $f(t)$ is a numerical term.

(iv) An expression is a numerical term just if it can be shown to be so on the basis of (i), (ii), (iii) above.

Functional terms are defined recursively as follows:

(i') Any function variable $f, g, \ldots$ and constants 0,1 are functional terms.

(ii') If $t_{1}, t_{2}$ are functional terms, then $\left(t_{1}+t_{2}\right),\left(t_{1}-t_{2}\right)$ are functional terms.

(iii') An expression is a functional term just if this follows from (i') and (ii').

Having defined the terms allowed by our small language, we now proceed to define the family of formulae comprising the extension of Tarski's theory of reals that we want to discuss. First of all we define 'atoms'. An atom is an expression of one of the following forms:

$$
\begin{aligned}
& t_{1}=t_{2}, \quad t_{1}<t_{2}, \quad n_{1}=n_{2}, \quad n_{1}<n_{2}, \quad u p\left(n_{1}, t_{1}, t_{2}\right),
\end{aligned}
$$

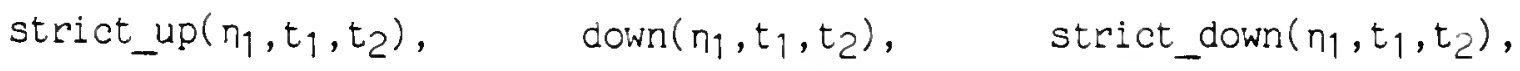




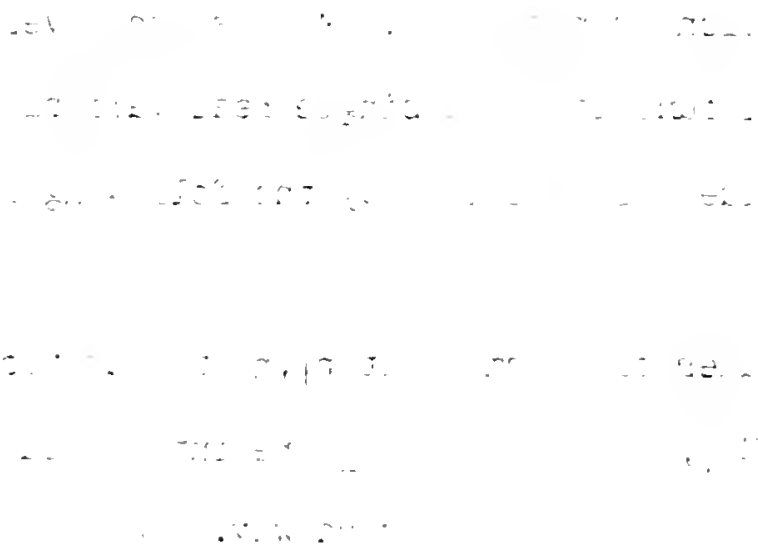


convex $\left(n_{1}, t_{1}, t_{2}\right)$, concave $\left(n_{1}, t_{1}, t_{2}\right)$, where $t_{1}, t_{2}$ are numerical terms and $\eta_{1}, n_{2}$ are functional terms. (The intended meaning of up, strict_up, etc. will be defined below.) Finally, we define a formula in the theory of Reals with Monotone and Convex Functions (RMCF) to be any boolean combination of atons.

Let $\phi$ be a formula in RMCE.

Definition 3.2. An interpretation $M$ of the symbols of $\phi$ is a real model of $\phi$ if:

(1) For any real variable $\mathrm{x}$, the value $\mathrm{Mx}$ associated to $\mathrm{x}$ is a real number.

(2) For any function variable $f, M f$ is an everywhere defined continuous real function of a single real variable.

(3) The truth value assigned to $\phi$ by the following rule is the value true:

Let $t_{1}, t_{2}$ be numerical terms, let $n_{1}, n_{2}$ be functional terms, and assume that $M t_{1}, M t_{2}, M n_{1}, M n_{2}$ are interpretations assigned to them by the model $M$ in the standard way. Then

(3a) $\quad t_{1}=t_{2}\left(\right.$ resp. $\left.t_{1}<t_{2}\right)$ is true if and only if $M t_{1}=M t_{2}\left(\operatorname{resp} . M t_{1}<M t_{2}\right)$

(3b) $n_{1}=n_{2}\left(r e s p . n_{1}<n_{2}\right)$ is true if and only if $M n_{1}=M n_{2}\left(\right.$ resp. $\left.M n_{1}<M n_{2}\right)$ pointwise

(3c) $\quad u p\left(n_{1}, t_{1}, t_{2}\right)$ (resp. strict_up $\left.\left(n_{1}, t_{1}, t_{2}\right)\right)$ is true if and only if $M t_{1} \geq M t_{2}$, or $M t_{1}<M t_{2}$ and the function $M n_{1}$ is monotone nondecreasing (resp. strictly increasing) in the intervai $\left[M t_{1}, M t_{2}\right]$.

(3d) convex $\left(\eta_{1}, t_{1}, t_{2}\right)$ is true if and only if $M t_{1} \geq M t_{2}$ or 


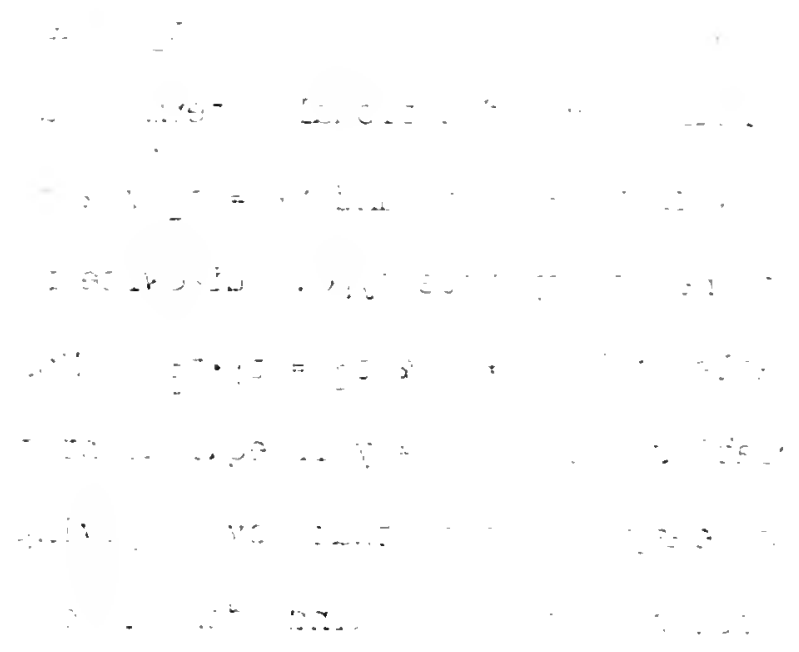


$M t_{1}<M t_{2}$ and the function $M n_{1}$ is convex in the interval $\left[M t_{1}, M t_{2}\right]$.

(3e) The truth values of down $\left(n_{1}, t_{1}, t_{2}\right)$, strict_down $\left(n_{1}, t_{1}, t_{2}\right)$ concave $\left(n_{1}, t_{1}, t_{2}\right)$ are defined in a manner completely analogous to the corresponding definitions (3c), (3d), with the sole difference that increasing and decreasing have to be interchanged and concave has to be read in place of convex.

Observe that formulae $t_{1}=t_{2}-t_{3}, t_{1}>t_{2}$ where $t_{1}, t_{2}, t_{3}$ are either numerical or functional terms are respectively equisatisfiable with $t_{2}=t_{1}+t_{3}$ and $t_{1}=t_{2}+v \& v>0$, where $v$ is a variable of the appropriate type. Likewise formula $t_{1}=t_{2} / t_{3}$ is equisatisfiable with $t_{3} \neq 0 \& t_{2}=t_{1} \cdot t_{3}$. Moreover if $x$ and $y$ are real variables, then $\mathrm{x} \neq \mathrm{y}$ is equivalent to $\mathrm{x}\langle\mathrm{y} v \mathrm{x}\rangle \mathrm{y}$ : Therefore it is easy to see that by applying an elementary normalization process of the kind described in the preceding section we can without loss of generality consider only sets of clauses each of which has one of the following types:

$\begin{array}{ll}(+) & x=y+z \\ (\cdot) & x=y \cdot z \\ (>) & x>0 \\ (f) & x=f(y) \\ (f+) & f=g+h \\ (f f) & f \neq g \\ (f>) & f>0 \\ (u-d) & \operatorname{up}(f, x, y), \quad \operatorname{down}(f, x, y)\end{array}$




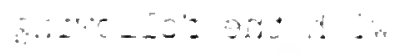

$$
\begin{aligned}
& \because \quad \neg \quad \therefore \quad-\cdots \\
& \because, \quad \therefore \because z \quad \therefore \quad \therefore \quad \therefore \quad \ldots \\
& \because 7 \quad \because 2 \cdots+\ldots-\ldots \\
& \text { L } \quad \ldots+\ldots i=i \\
& \ldots \div \div, \cdots+\cdots \\
& \therefore \quad \cdots+5
\end{aligned}
$$


(strict) strict_up $(f, x, y), \quad \operatorname{strict} d o w n(f, x, y)$

$(-u-d)-|\operatorname{up}(f, x, y),-| \operatorname{down}(f, x, y)$

(- strict) - strict_up $(\mathrm{f}, \mathrm{x}, \mathrm{y}), \overline{\mid} \operatorname{strict} \operatorname{down}(\mathrm{f}, \mathrm{x}$,

(con) convex $(f, x, y), \operatorname{concave}(f, x, y)$

$(\mid c o n)-|\operatorname{convex}(f, x, y),-|$ concave $(f, x, y)$,

where $x, y, z$ are real variables and $f, g, h$ are function variables,

In order to show that the theory RMCF is decidable, we will exhibit a simple reduction that can be applied to a given initial formula, and that yields a formula with the following properties:

(a) no function variable occurs in the resulting formula (therefore in particular only clauses of the types $(+, \cdot,>$ ) can occur);

(b) the initial and the resulting formulae are equisatisfiable.

Note that (a) and (b) combined with the basic result that formulae involving only clause of the types $(+, \cdot\rangle$,$) are decidable (see [Tar51],$ [Col75]) implies the decidability of the theory RMCF.

To do this, let $\phi$ be a formula of RMCF, which by Lemma 2.4 and by the foregoing discussion can be assumed to be a conjunction of clauses of the types $(+)-(-\mid$ con $)$ listed above. We begin by treating all clauses containing a negation. The reason for this is that any negative clause involving a function symbol (except as a numerical term; see Definition 3.1) can be viewed as an implicit existential. assertion whose quantifier is easy to eliminate by adding all instantiation of the quantified body to our set of clauses. More specifically, we proceed as follows:

Simplification Step 1:

(1a) For any literal $f \neq g$ occurring in $\phi$, introduce three new 


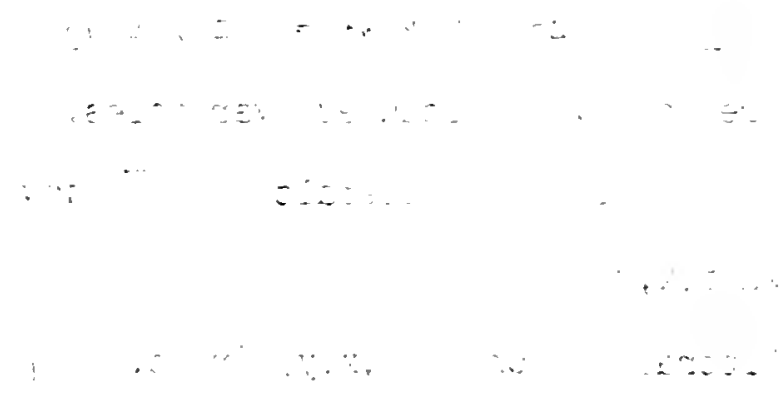


variables $x_{1}, x_{2}, y$ and replace $f \neq g$ by the formula $x_{1}=f(y)$ \& $x_{2}=g(y) \& x_{1} \neq x_{2}$ (as previously noted, we can use $x_{1}>x_{2} v$ $\mathrm{x}_{2}>\mathrm{x}_{1}$ in place of $\mathrm{x}_{1} \neq \mathrm{x}_{2}$ but for reasons of clarity we do not insist on noting such simplifications at every step of what follows).

(1b) Replace any literal $\mid u p(f, x, y)$ (resp. - strict_up(f,x,y)) in $\phi$ by a formul.

$A \& w_{1}>w_{2} \quad\left(\right.$ resp. $\left.A \& w_{1} \geq w_{2}\right)$,

where $A \equiv x \leq z_{1}<z_{2} \leq y \& w_{1}=f\left(z_{1}\right) \& w_{2}=f\left(z_{2}\right)$ and where $z_{1}, z_{2}, w_{1}, w_{2}$ are newly introduced variables. Make analogous substitutions for literals $\quad-$ down $(f, x, y)$ and - strict_down $(f, x, y)$.

(1c) Replace any literal $-j \operatorname{convex}(f, x, y)$ (resp. $-\mid$ concave $(f, x, y)$ ) by the formula

$B \&\left(w_{2}-w_{1}\right)\left(z_{3}-z_{1}\right)>\left(z_{2}-z_{1}\right)\left(w_{3}-w_{1}\right)$

(resp. B \& $\left.\left(w_{2}-w_{1}\right)\left(z_{3}-z_{1}\right)<\left(z_{2}-z_{1}\right)\left(w_{3}-w_{1}\right)\right)$

where

$B \equiv x \leq z_{1}<z_{2}<z_{3} \leq y \& w_{1}=f\left(z_{1}\right) \& w_{2}=f\left(z_{2}\right) \& w_{3}=f\left(z_{3}\right)$

and where $z_{1}, z_{2}, z_{3}, \quad w_{1}, w_{2}, w_{3}$ are newly introduced real variables. (The formula above just expresses the existence of three points $z_{1}, z_{2}, z_{3}$, such that $\left(z_{2}, f\left(z_{2}\right)\right.$ ) lies above (resp. below) the straight Iine joining the two points $\left(z_{1}, f\left(z_{1}\right)\right)$ and $\left(z_{3}, f\left(z_{3}\right)\right)$ 


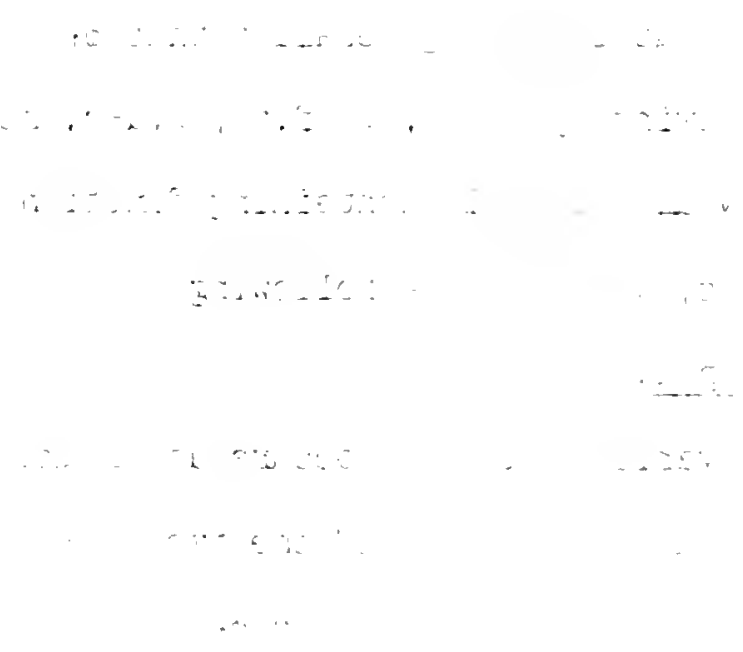


Let $\phi_{1}$ be the resulting formula; then it is easy to see that $\phi$ and $\phi_{1}$ are equisatisfiable. Indeed clearly any model of $\phi$ is a model of $\phi_{1}$. Conversely let $M$ be a model of $\phi_{1}$. Then it follows easily, e.g., that if $\mathrm{f} \neq \mathrm{g}$ is in $\phi$ then $\mathrm{Mf} \neq \mathrm{Mg}$. In fact by step (1a) a formula $\mathrm{x}_{1}$ $=f(y) \& x_{2}=g(y) \& x_{1} \neq x_{2}$ is present in $\phi_{1}$. Therefore since $M$ is a model for $\phi$ we have $M x_{1}=(M f)(M y), M x_{2}=(M g)(M y)$ and $M x_{1} \neq M x_{2}$, that is (Mf) (My) $\neq(\mathrm{Mg})(\mathrm{My})$, which in particular shows that (Mf) $\neq(\mathrm{Mg})$. It can be established in much the same way that steps (1b) and (1c) do not disrupt satisfiability of $\phi$. Normalizing $\phi_{1}$ again and using Lemma 2.4, we can assume without loss of generality that $\phi_{1}$ is a conjunction of literals of the following types $(+, \cdot,>, f, f+, f>, u-d$, strict, con).

We now show how all literals containing function variables can be eliminated. For this, we apply the following

\section{Preparatory Step 2:}

For any real variable $\mathrm{x}$ which occurs as argument of any function variable or as a bound in a literal of the type ( $u-d$, strict, con) and for any function variable $f$ occurring in $\phi_{1}$, introduce a new variable $z_{x, f}$ and add the formula $z_{x, f}=f(x)$ to $\phi_{1}$, unless a literal $y=f(x)$ exists already.

Let $\phi_{2}$ be the resulting formula. Since each newly introduced variable $z_{X}, f$ appears in just one equality literal, it is clear that $\phi_{1}$ and $\phi_{2}$ are still equisatisfiable at this stage. Note that Step 2 is applied simply in preparation for the elimination of all (u-d, strict, con) clauses; it serves only to ensure that all potentially significant implicit function evaluations appear explicitly. We are now ready for step 3. 


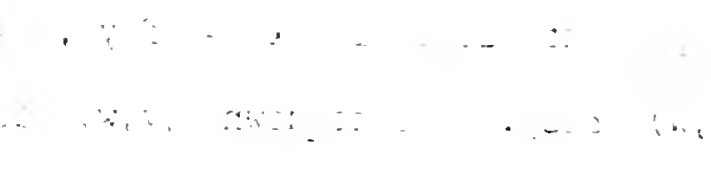

$$
\begin{aligned}
& -\quad \because \because \because . c= \\
& \therefore ?=+\therefore-\cdots+=\square
\end{aligned}
$$


Simplification Step 3

(3a) For each quadruple of literals $x=f(y), x^{\prime}=g(y), x^{\prime \prime}=h(y)$, $f=g+h$, add $x=x^{\prime}+x^{\prime \prime}$.

(3b) For each pair of literals $x=f(y), f>0$ add $x>0$.

(3c) For each pair of literals $x=f\left(y_{1}\right), x^{\prime}=f\left(y_{2}\right)$ add the formula $\mathrm{y}_{1}=\mathrm{y}_{2} \rightarrow \mathrm{x}=\mathrm{x}^{\prime}$.

(3d) For each triple of literals $x=f(y), x^{\prime}=f\left(y^{\prime}\right), u p(f, v, w)$ (resp. down $(f, v, w)$ ) add the formula $v \leq y<y^{\prime} \leq w \rightarrow x \leq x^{\prime}$ (resp. $\mathrm{v} \leq \mathrm{y}<\mathrm{y}^{\prime} \leq \mathrm{w} \rightarrow \mathrm{x} \geq \mathrm{x}^{\prime}$ )

(3e) For each triple of literals $x=f(y), x^{\prime}=f^{\prime}\left(y^{\prime}\right)$, and strict_up $(f, v, w)$ (resp. strict_down $(f, v, w)$ ) add the formula

$$
v \leq y<y^{\prime} \leq w \rightarrow x<x^{\prime} \quad\left(\text { resp. } v \leq y<y^{\prime} \leq w \rightarrow x>x^{\prime}\right)
$$

(3f) For each quadruple of literals $x=f(y), x^{\prime}=f(y), x^{\prime \prime}=f\left(y^{\prime \prime}\right)$, convex $(f, v, w)$ (resp. concave $(f, v, w)$ ) add the formula:

$$
v \leq y^{\prime}<y<y^{\prime \prime} \leq w \rightarrow\left(y-y^{\prime}\right) \cdot\left(x^{\prime \prime}-x^{\prime}\right) \geq\left(x-x^{\prime}\right) \cdot\left(y^{\prime \prime}-y^{\prime}\right)
$$

(resp. $\left.\quad v \leq y^{\prime}<y<y^{\prime \prime} \leq w \rightarrow\left(y-y^{\prime}\right) \cdot\left(x^{\prime \prime}-x^{\prime}\right) \leq\left(x-x^{\prime}\right) \cdot\left(y^{\prime \prime}-y^{\prime}\right)\right)$. (Observe that the implication above says just that the 'point' $(y, x)$ is above (resp. under) the straight line which passes through the 'points' $\left(y^{\prime}, x^{\prime}\right)$ and $\left(y^{\prime \prime}, x^{\prime \prime}\right)$ in a plane-coordinate.)

(3g) Drop all literals involving any function variable.

Let $\phi_{3}$ be the formula obtained by execution of step 3 . Then $\phi_{3}$ is a formula involving only real variables, the arithmetic operators +, -, - and the predicates $=\langle, \leq$, i.e. is a function of the polynomial 


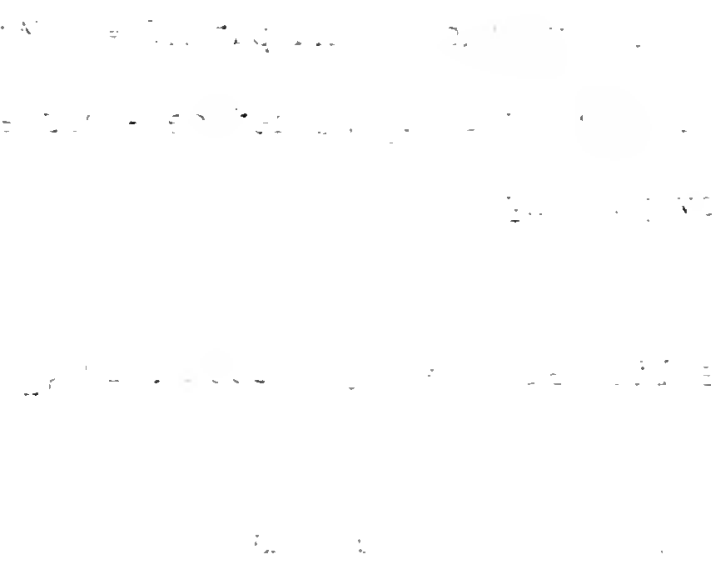


theory of reals, which is decidable by Tarski's famous result [Tar51 j. Thus to prove that the theory RMCF is decidable we only need to show that $\phi_{2}$ and $\phi_{3}$ are equisatisfiable, which we do as follows.

First of all, since all formulae introduced by step 3 are consequences of $\phi_{2}$, any model of $\phi_{2}$ is also a model of $\phi_{3}$.

Conversely let $M$ be a model of $\phi_{3}$. We will show how to extend $M$ in a manner allowing all function variables to be interpreted in such a way as to satisfy all clauses of type ( $f, f+, f>0, u-d$, strict,con). Also, for this, let $V$ be the set of all real variables occurring in $\phi_{2}$ as arguments of some function variable, and put $M V=\{M y \mid y \in V\}$. For any function variable $f$ occurring in $\phi_{2}$ we define a corresponding function F having domain MV, by putting

(1) $F(M y)=M x$ if a literal $x=f(y)$ occurs in $\phi_{2}$.

The following easy lemma gives us what we now need.

Lemma 3.3. For any function variable $f$ in $\phi_{2}$, the corresponding function $F$ defined by (1) is single valued and has domain MV.

Proof: The only reason for which $F$ could fail to be single valued is that $\phi_{2}$ contains two literals $x=f(y), x^{\prime}=f^{\prime}\left(y^{\prime}\right)$, while $M y=M^{\prime}$ and $M x \neq M x^{\prime}$. But this is impossible, since in such a case step (3c) would have introduced a literal $y=y^{\prime}+x=x^{\prime}$, which force $M x=M x^{\prime}$. Moreover, as observed just after the text of step 2, all function variables are evaluated in the same set of real variables, therefore it follows tinat the domain of any function $E$ is just $M V$.

It just remains to extend our model functions $F$ to the whole real axis. This can be done by the obvious procedure of linear 
..

$$
\therefore-\cdots+\ldots
$$

$$
\begin{aligned}
& \text { (n) } \cdots+\cdots \\
& +\quad+\cdots+\infty \\
& =\quad \therefore \quad \cdots=\ldots
\end{aligned}
$$

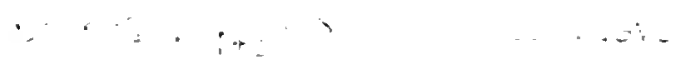

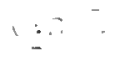


interpolation. More specifically let $n_{1}, n_{2}, \ldots, n_{r}$ be the elements of MV, taken in increasing order. Then for any real $n$ we put
$\left(F\left(n_{1}\right)\right.$
if $n<n_{1}$
$\begin{aligned} \text { (2) }(\text { Mf })(n)=\frac{n-n_{i}}{n_{i+1}-n_{i}}\left(F\left(n_{i}+1\right)-F\left(n_{i}\right)\right)+F\left(n_{i}\right) & \text { if } n_{i} \leq n<n_{i}+1, \quad i \varepsilon\{1, \ldots, r-1\} \\ F\left(n_{r}\right) & \text { if } n_{r} \leq n\end{aligned}$

Then for any function variable $f$ in $\phi_{2}$, Mf is a continuous function. Moreover, it follows at once by (1) and (2) that all clauses of type (f) in $\phi_{2}$ are correctly modeled. We show now that clauses of type $(f+, f>, u-d$, strict,con) are also satisfied.

For this, let $\mathrm{f}=\mathrm{g}+\mathrm{h}$ be a literal of $\phi_{2}$. We want to show that for any real $n$, $(M f)(n)=(M g)(n)+(M h)(n)$. But this is clear if either $n<n_{1}$, or $n>n_{r}$, or $n=n_{i}$ for some $i=1, \ldots, r$, by (1), (2) and step (3a). Moreover, if $n_{i}<n<n_{i+1}$, for some $i=1, \ldots, r$, then

$$
\begin{aligned}
(\text { Mf })(n) & =\frac{\eta-n_{i}}{n_{i+1}-n_{i}}\left(F\left(n_{i+1}\right)-F\left(n_{i}\right)\right)+F\left(n_{i}\right) \\
& =\frac{n-n_{i}}{n_{i+1}-n_{i}}\left(G\left(n_{i+1}\right)+H\left(n_{i+1}\right)-G\left(n_{i}\right)-H\left(n_{i}\right)\right)+G\left(n_{i}\right)+H\left(n_{i}\right) \\
& =\frac{n-n_{i}}{n_{i}+1-n_{i}}\left(G\left(n_{i+1}\right)-G\left(n_{i}\right)\right)+G\left(n_{i}\right)+\frac{n-n_{i}}{n_{i+1}-n_{i}}\left(H\left(n_{i+1}\right)-H\left(n_{i}\right)\right)+H\left(n_{i}\right) \\
& =(M \delta)(n)+(M h)(n) .
\end{aligned}
$$

This establishes that every clause $f=g+h$ is satisfied. It is also clear by (1), (2) and step (3b) that any literal $\mathrm{f}>0$ is correctly modeled. By (1), (2) and in view of the clauses added in steps (3d) and ( $3 e$ ) it follows easily that literals (u-d,strict) are also satisfied. Finally, let convex $(f, x, y)$ be a clause of $\phi_{2}$. If $M x \geq M y$, then $M$ satisfies the clause convex $(f, x, y)$ which is void. Otherwise $M x$ $<M y$; let $M x=\eta_{i}$ and $M y=\eta_{j}$, with $i<j, i, j \varepsilon\{1, \ldots, r\}$. If $M f$ 


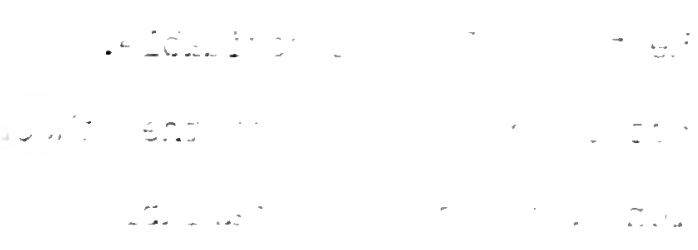


coincides just with the straight line passing through the points $\left(\eta_{i},(M f)\left(n_{i}\right)\right)$ and $\left(\eta_{j},(M f)\left(n_{j}\right)\right)$, then clearly Mf is convex (strict convexity is not required!) in the interval $\left[n_{i}, n_{j}\right]$. On the other hand if $\mathrm{Mf}$ is not a straight $I$ ine in the interval $\left[\eta_{i}, \eta_{j}\right]$, then by step (3f) the graph of Mf is the union of convex pieces. It follows therefore by an elementary argument that $\mathrm{Mf}$ is convex in the interval $\left[n_{i}, n_{j}\right]$. Thus in both cases $M f$ is convex in the interval [MX, My], which shows that $M$ satisfies the clause convex $(f, x, y)$. Since $M$ satisfies all purely arithmetic clauses in $\phi_{2}$ of type $\left.(*, \cdot\rangle,\right)$, it follows at once that $M$ is a model of $\phi_{2}$. Q.E.D.

This completes the proof of

Theorem 3.4. The theory RMCF is decidable.

Remark. Note that the restriction of the theory RMCF in which neither multiplication between real variables, nor the predicates 'convex' and 'concave' are allowed can be decided more efficiently by application of a linear programing algorithm ([Dan63]). The reason why a 'convex' and 'concave' predicate are not allowed in this simple case is that using these predicates and 'strict up' also it is possible to express the assertion that a function is a strictly increasing straight line, and hence it is an easy matter to express the product relationship among real variables. Hence a theory allowing convexicy to be asserted along with the other predicates we have listed can be used to express any relationship admitted in Tarski arithmetic, making it plain that no such theory can admit a decision algorithm as efficient as simple linear programming. 


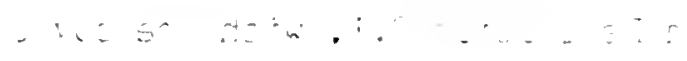




4. 'Extended Tarski' theory of reals with multivariate continuous functions.

For the sake of simplicity, we will only consider the case in which all function variables have the same arity n. The general case, that is when functions having different arity are allowed, can be easily reduced to this simpler theory by an obvious treatment by cases. Again we allow two kinds of variable, namely real variables $x, y, \ldots$, and n-ary real function variables $f, 8, \ldots$. Constants 0,1 , both numerical and functional, are also allowed. Numerical and functional terms are defined as in Definition 3.1, with the obvious replacement of condition (iii) by

(ii $\left.i_{1}\right)$ If $t_{1}, \ldots, t_{n}$ are numerical terms and $f$ is a function variable, then $f\left(t_{1}, \ldots, t_{n}\right)$ is a numerical term.

We define the family of formulae of the theory of reals with monotone multivariate continuous functions (RMMCF) as follows. An atom is an expression of one of the following forms: $t_{1}=t_{2}, t_{1}<t_{2}, n_{1}=n_{2}$, $\eta_{1}<n_{2}, u p\left(n_{1} ; t_{1}, u_{1} ; \ldots ; t_{n}, u_{n}\right)$, strict_up $\left(n_{1} ; t_{1}, u_{1} ; \ldots ; t_{n}, u_{n}\right)$, where $t_{1}, t_{2}, \ldots, t_{n}, u_{1}, \ldots, u_{n}$ are numerical terms, and $n_{1}, n_{2}$ are functional terms. (Observe that no predicate 'convex' or 'concave' is allowed in this theory.) A formula in RMMCF is defined to be any Boolean combination of atoms. The intended meaning of the predicates up, strict_up, etc. which appear above is defined as follows. Let $\phi$ be a formula in RMMCF. 


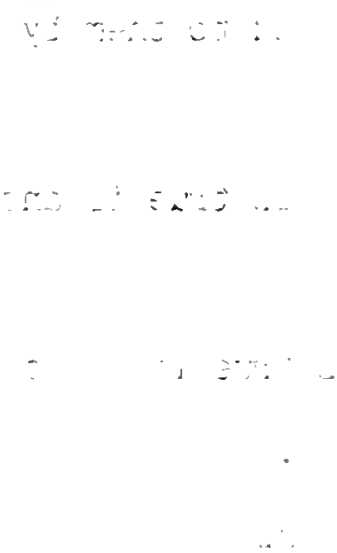


Definition 4.1. An interpretation $M$ of the symbols of $\phi$ is a model of $\phi$ if:

(1) For any real variable $\mathrm{x}$, the value $\mathrm{Mx}$ associated to $\mathrm{x}$ is a real number.

(2) For any function variable $\mathrm{f}$, $\mathrm{Mf}$ is an everywhere defined $\mathrm{n}$-ary continuous real function.

(3) The truth value assigned to $\phi$ by the following rule is the value true:

Let $t_{1}, t_{2}, \ldots, t_{n}, u_{1}, \ldots, u_{n}$ be numerical terms and $n_{1}, n_{2}$ be functional terms, and assume that $M t_{1}, \ldots, M t_{n}, M u_{1}, \ldots, M u_{n}, M n_{1}$, $M n_{2}$ are the interpretations assigned to them by the model $M$ in the standard way. Then

(3a) $t_{1}=t_{2}$ (resp. $t_{1}<t_{2}$ ) is true if and only if $M t_{1}=M t_{2}$ (resp. $\left.M t_{1}<M t_{2}\right)$.

(3b) $n_{1}=n_{2}$ (resp. $n_{1}<n_{2}$ ) is true if and only if $M n_{1}=M n_{2}$ (resp. $\left.M n_{1}<M n_{2}\right)$ pointwise.

(3c) up $\left(\eta_{1} ; t_{1}, u_{1} ; \ldots ; t_{n}, u_{n}\right) \quad$ (resp. strict_up $\left(\eta_{1} ; t_{1}, u_{1} ; \ldots ;\right.$ $\left.\left.t_{n}, u_{n}\right)\right)$ is true if and only if for every $\left(\alpha_{1}, \ldots, \alpha_{n}\right)$, $\left(B_{1}, \ldots, B_{n}\right) \varepsilon\left[M t_{1}, M u_{1}\right] \times \ldots \times\left[M t_{n}, M u_{n}\right]$, with $\alpha_{i} \leq \beta_{i}$ for all $i=1, \ldots, n$, and $\alpha_{j}<B_{j}$ for some $j \varepsilon\{1, \ldots, n\}$, $\left(M_{n_{1}}\right)\left(\alpha_{1}, \ldots, \alpha_{n}\right) \leq\left(M_{n_{1}}\right)\left(\beta_{1}, \ldots, \beta_{n}\right)\left(\operatorname{resp} . \quad\left(M_{n_{1}}\right)\left(\alpha_{1}, \ldots, \alpha_{n}\right)\right.$ $\left.<\left(M_{1}\right)\left(\beta_{1}, \ldots, \beta_{n}\right)\right)$.

By applying a normalization process of the kind described in the preceding section, we can reduce the satisfiability problem for the theory RMMCF to the satisfiability problem for conjunctions of liter...3 of the following types:

$(+) \quad x=y+z$ 
$\therefore i$

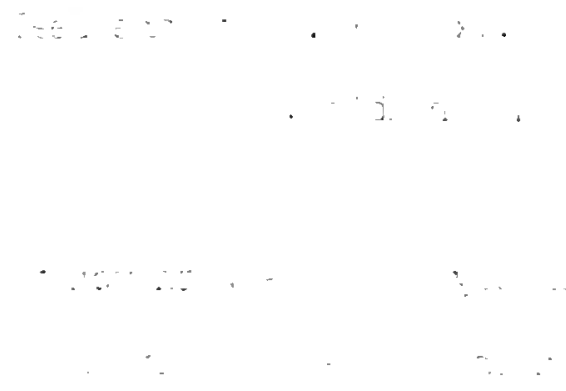



(.) $\quad x=y \cdot z$
(>) $x>0$
(f) $x=f\left(y_{1}, \ldots, y_{n}\right)$
$(\mathrm{f}+) \quad f=g+h$
$(f \neq) \quad f \neq g$
(f $>$ f $>0$
(up) $u p\left(f ; x_{1}, y_{1} ; \ldots ; x_{n}, y_{n}\right)$
(strict_up) strict_up $\left(f ; x_{1}, y_{1}, ; \ldots ; x_{n}, y_{n}\right)$
(† up $\rceil_{\text {up }\left(f ; x_{1}, y_{1} ; \ldots ; x_{n}, y_{n}\right)}$
(-|strict_up) Tstrict_up $\left(f ; x_{1}, y_{1} ; \ldots ; x_{n}, y_{n}\right)$

where $x, y, z, x_{1}, \ldots, x_{n}, y_{1}, \ldots, y_{n}$ are real variables, and $f, g, h$ are function variables.

In analogy with the theory of reals with monotone and convex continuous functions discussed in the preceding section, we will prove that the theory RMMCF is decidable by showing how a conjunction of literals of the types (+) - ( $\mid$ strict_up) listed above can be reduced to a conjunction involving only literals $(+, \cdot,<)$. Tarski's result concerning the real field (see [Tar51]) will then imply the decidability of RMMCE.

Let $\phi$ be a conjunction of literals each of which is of one of the types (+) - ( / strict up) above. We apply the following simplification steps to $\varphi$, to eliminate all literals other than those of type $(+, \cdot,<)$. 





Simplification Step 1.

(1a) For any literal $f \neq g$ occurring in $\phi$, introduce new variables $x_{1}, x_{2}, y_{1}, \ldots, y_{n}$ and replace $f \neq \&$ by the formula $x_{1}=$ $f\left(y_{1}, \ldots, y_{n}\right) \& x_{2}=g\left(y_{1}, \ldots, y_{2}\right) \& x_{1} \neq x_{2}$.

(1b) Replace any literal $-u p\left(f ; x_{1}, y_{1} ; \ldots ; x_{n}, y_{n}\right.$ ) (resp. I strict_up $\left.\left(f ; x_{1}, y_{1} ; \ldots ; x_{n}, y_{n}\right)\right)$ by the formula

$$
A \& u_{1}>u_{2} \quad\left(\text { resp. } A \& u_{1} \geq u_{2}\right) \text {, }
$$

$$
\text { where } A \begin{aligned}
& \sum_{i=1}^{n}\left(x_{i} \leq w_{i} \leq z_{i} \leq y_{i}\right) \&{ }_{i=1}^{n}\left(w_{i}<z_{i}\right) \\
& \& u_{1}=f\left(w_{1}, \ldots, w_{n}\right) \& u_{2}=f\left(z_{1}, \ldots, z_{n}\right)
\end{aligned}
$$

and where $w_{1}, \ldots, w_{n}, z_{1}, \ldots, z_{n}, u_{1}, u_{2}$ are newly introduced variables. (Note that the formulae above simply express the fact that the interval $\left[x_{1}, y_{1}\right] \times \ldots \times\left[x_{n}, y_{n}\right]$ contains two points $\left(w_{1}, \ldots, w_{n}\right),\left(z_{1}, \ldots, z_{n}\right)$ which falsify the predicate $\mathrm{up}\left(\mathrm{f} ; \mathrm{x}_{1}, \mathrm{y}_{1} ; \ldots ; \mathrm{x}_{\mathrm{n}}, \mathrm{y}_{\mathrm{n}}\right)$ (resp. strict_up(f; $\mathrm{x}_{1}, \mathrm{y}_{1} ; \ldots$; $\left.x_{n}, y_{n}\right)$ ) according to (3c) of Definition 4.1.)

Let $\phi_{1}$ be the formula into which $\phi$ is transformed by application of this simplification step. It is clear that $\phi$ and $\phi_{1}$ are equisatisfiable. It therefore follows that $\phi_{1}$ can be taken to be a conjunction of literals of the following types $(+, \cdot,>, f, f+, f>$, up, strict_up). Simplification Step 1 eliminates literals of type ( $f \neq$, - up, (strict_up). To eliminate the remaining clauses involving function variables, namely those of type ( $f, f+, f>$, up, strict_up), we then subject $\phi_{1}$ to the following 


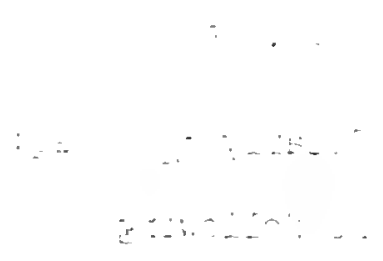


Simplification Step 2.

For every $i=1, \ldots, n$, let $v_{i}$ be the set of real variables which occur either as the $i$-th argument of some function variable in $\phi_{1}$ or as left or right $(i+1)$-st argument of a predicate of type up, strict_up in $\phi_{1}$. In other words, $V_{i}$ is the set of all variables which take values in the $i$-th factor space of the product space $\mathbb{B}^{n}$. For any $\xi=$ $\left(\xi_{1}, \ldots, \xi_{n}\right) \varepsilon V_{1} \times \ldots \times V_{n}$ and any function variable $f$ occurring in $\phi_{1}$, introduce a new real variable $z_{f, \xi}$, and add the formula $z_{f, \xi}=$ $f\left(\xi_{1}, \ldots, \xi_{n}\right)$, unless a literal y $=f\left(\xi_{1}, \ldots, \xi_{n}\right)$ exists already.

Call the resulting formula $\phi_{2}$. Clearly $\phi_{2}$ is equisatisfiable with $\phi_{1}$. Once this has been done, apply the following

Simplification Step 3.

(3a) For each quadruple of literals $x=f\left(y_{1}, \ldots, y_{n}\right), x^{\prime}=$ $g\left(y_{1}, \ldots, y_{n}\right), x^{\prime \prime}=h\left(y_{1}, \ldots, y_{n}\right), f=g+h$, add $x=x^{\prime}+x^{\prime \prime}$.

(3b) For each pair of literals $x=f\left(y_{1}, \ldots, y_{n}\right), f>0$, add $x>0$.

(3c) For each pair of literals $x=f\left(y_{1}, \ldots, y_{n}\right), x^{\prime}=f\left(y, \ldots, y_{n}^{\prime}\right)$ add the formula

$$
\sum_{i=1}^{n}\left(y_{i}=y_{i}^{\prime}\right) \rightarrow x=x^{\prime} .
$$

(3d) For each triple of literals $x=f\left(y_{1}, \ldots, y_{n}\right), x^{\prime}=f\left(y \nmid, \ldots, y_{n}^{\prime}\right)$, $u p\left(f ; v_{1}, w_{1} ; \ldots ; v_{n}, w_{n}\right)$ (resp. strict_up $\left.\left(f ; v_{1}, w_{1} ; \ldots ; v_{n}, w_{n}\right)\right)$ add the formula

$$
B \rightarrow x \leq x^{\prime} \quad\left(\text { resp. } B \rightarrow x<x^{\prime}\right)
$$




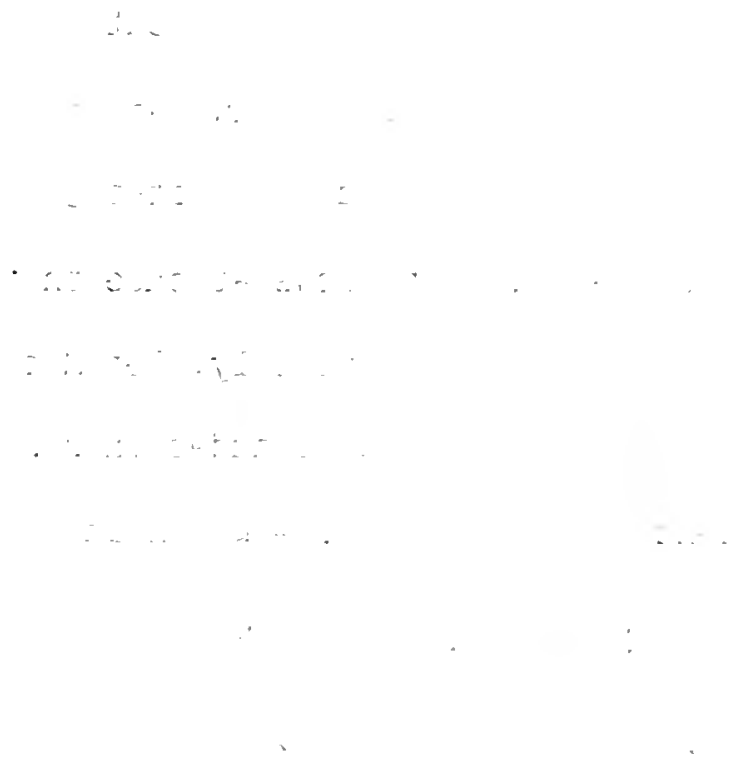


where $B \equiv \sum_{i=1}^{n}\left(v_{i} \leq y_{i} \leq y_{i} \leq w_{i}\right) \& \underset{i=1}{n}\left(y_{i}<y_{i}^{i}\right)$.

(3e) From $\phi_{2}$, drop all literals involving any function variable.

Let $\phi_{3}$ be the formula produced by Simplification Step 3. Observe that $\Phi_{3}$ is a formula of the polynomial theory of reals, which is known to be decidable $[\operatorname{Tar} 51]$. Therefore in order to prove the decidability of the theory RMMCF, it suffices to show that Simplification Step 3 preserves satisfiability, namely that $\phi_{2}$ and $\phi_{3}$ are equisatisfiable. This can be done as follows. Since all formulae added by step 3 are consequences of $\Phi_{2}$ (cfr. Definition 4.1), it follows at once that any model of $\phi_{2}$ can be extended to a model of $\phi_{3}$. Conversely, let $M$ be a model of $\phi_{3}$.

It follows in virtue of the formulae added in simplification steps 2 and 3 that the presence of a formula $f=g+h$ in $\phi$ implies that

$$
\text { (Mf) }\left(u_{1}, \ldots, u_{n}\right)=(M g)\left(u_{1}, \ldots, u_{n}\right)+(M h)\left(u_{1}, \ldots, u_{n}\right)
$$

for all $\left(u_{1}, \ldots, u_{n}\right) \subseteq D$, where $D=M V_{1} \times \ldots \times M V_{n}$. Similarly, the presence of a formula $f>0$ in $\phi$ implies that $(M f)\left(u_{1}, \ldots, u_{n}\right)>0$ for all $\left(u_{1}, \ldots, u_{n}\right) \quad \varepsilon \quad D ;$ and if $u p\left(f ; x_{1}, y_{1} ; \ldots ; x_{n}, y_{n}\right)$ (resp. strict_up $\left.\left(f ; x_{1}, y_{1} ; \ldots ; x_{n}, y_{n}\right)\right)$ belongs to $\phi$, then $(M f)\left(u_{1}, \ldots, u_{n}\right) \geq$ $(M f)\left(v_{1}, \ldots, v_{n}\right) \quad\left(\right.$ resp. $\left.(M f)\left(u_{1}, \ldots, u_{n}\right)>(M f)\left(v_{1}, \ldots, v_{n}\right)\right)$ for any two distinct $\left(u_{1}, \ldots, u_{n}\right) \in D,\left(v_{1}, \ldots, v_{n}\right) \in D$ such that $M y_{i} \geqq u_{i} \geqslant v_{i} \geqq M x_{i}$ for all $i=1, \ldots, n$.

The fact that $\phi_{2}$ and $\phi_{3}$ are equisatisfiable will therefore follow immediately from the following elementary extension lemma.

Lemma 4.2. For each $i=1, \ldots, n$ let $u(i)<\ldots<u^{(i)}{ }_{m_{i}}$ be a finite, strictly increasing sequence of real numbers. Let $D$ be the product set 
$\because \cdots, \because \ldots$

$\cdots \quad \cdots \cdots$

$\because=\because \because \ldots$

-. 


$$
D=\left\{u\{1), \ldots, u_{m_{1}}^{(1)}\right\} \times \ldots \times\left\{u\left\{(n), \ldots, u_{m_{n}}^{(n)}\right\}\right.
$$

Let $\Phi$ be the space of all real valued functions defined on $D$. Then there exists a systematic procedure for extending each of the functions $f \varepsilon \phi$ to a continuous real-valued function $\mathrm{Ef}$ defined on all of $\mathrm{R}^{\mathrm{n}}$, which is such that $E(f+g)=E f+E g$, and $(E f)\left(x_{1} \ldots, x_{n}\right) \geq$ (Eg) $\left(x_{1}, \ldots, x_{n}\right)\left(\right.$ resp. $\left.\quad(E f)\left(x_{1}, \ldots, x_{n}\right)>(E g)\left(x_{1}, \ldots, x_{n}\right)\right)$ for all $\left(x_{1}, \ldots, x_{n}\right) \quad \varepsilon \quad R^{n}$ whenever $f\left(u_{1}, \ldots, u_{n}\right) \geq g\left(u_{1}, \ldots, u_{n}\right)$ (resp. $\left.f\left(u_{1}, \ldots, u_{n}\right)>g\left(u_{1}, \ldots, u_{n}\right)\right)$ for all $\left(u_{1}, \ldots, u_{n}\right) \varepsilon D$. Moreover, if $\left(X_{1}, \ldots, X_{n}\right) \in D,\left(Y_{1}, \ldots, Y_{n}\right) \in D$ and $f$ satisfies $f\left(u_{1}, \ldots, u_{n}\right) \geq$ $f\left(v_{1}, \ldots, v_{n}\right)$ (resp. $\left.f\left(u_{1}, \ldots, u_{n}\right)>f\left(v_{1}, \ldots, v_{n}\right)\right)$ for any two distinct $\left(u_{1}, \ldots, u_{n}\right) \& D, \quad\left(v_{1}, \ldots, v_{n}\right) \& D$ such that $Y_{i} \geq u_{i} \geq v_{i} \geq X_{i}$ for all $i=1, \ldots, n$, then Ef satisfies $(E f)\left(y_{1}, \ldots, y_{n}\right) \geq\left(E_{f}\right)\left(x_{1}, \ldots, x_{n}\right)$ (resp. $\left.(E f)\left(y_{1}, \ldots, y_{n}\right)>(E f)\left(x_{1}, \ldots, x_{n}\right)\right)$ for any two distinct $\left(y_{1}, \ldots, y_{n}\right) \varepsilon R^{n},\left(x_{1}, \ldots, x_{n}\right) \in R^{n}$ such that $Y_{i} \geq y_{i} \geq x_{i} \geq x_{i}$, $i=1, \ldots, n$.

Proof: We proceed by induction on the dimension $n$. Suppose that $\mathrm{n}=1$. Then $\mathrm{E}_{1} \mathrm{f}$ can be defined as the function which is linear in every interval between two points $u_{1}^{(1)}, u_{1+1}^{(1)}$ and which agrees with $f$ at each of the points $u_{1}^{(1)}$. It is also appropriate to define $E f$ to be constant in the two semi-infinite intervals $x \leq u(1)$ and $x \geq u_{m_{1}}^{(1)}$.

This same definition can be applied to the space of functions $f$ with values in any partially ordered vector space, and, in particular, to functions $f$ whose values belong to the space of continuous real-valued functions of $n-1$ variables $g\left(u_{2}, \ldots, u_{n}\right)$. In all such cases we have $E_{1}(\hat{f}+g)=E_{1} f+E_{1} g$ and $E_{1} f \geq E_{1} g$ (resp. $E_{1} f>E_{1} g$ ) at every point of the real axis if $f \geq g$ (resp. $f>g$ ) at every point of $i$ ts 




$$
\begin{aligned}
& \therefore=\sin y, \quad \because \cdots
\end{aligned}
$$


finite domain. Let $T$ be the transformation which maps each function $f\left(u_{1}, \ldots, u_{n}\right)$ defined on $D$ into the vector-valued function (Tf) (up $)$ defined by

$$
(T f)\left(u_{1}\right)\left(u_{2}, \ldots, u_{n}\right)=f\left(u_{1}, \ldots, u_{n}\right) ;
$$

here it should be understood that the values $(\mathrm{If})\left(\mathrm{u}_{1}\right)$ lie in the space $E_{n-1}$ of real-valued functions on the domain

$$
D_{2}=\left\{u\{2), \ldots, u_{m_{2}}^{(2)}\right\} \times \ldots \times\left\{u\{n), \ldots, u_{m_{n}}^{(n)}\right\}
$$

Let $E_{n-1}$ be the inductively def ined extension operator which transforms functions in $E_{n-1}$ into real-valued functions defined on all of $R^{n-1}$, and put

$$
\left(E_{n} f\right)=E_{1}\left(E_{n-1}(\operatorname{Tf}(\cdot))\right)
$$

where $E_{n-1}(\operatorname{Tf}(\cdot))$ simply denotes the function whose value at $u_{1}$ is $E_{n-1}\left(\operatorname{Tf}\left(L_{1}\right)\right)$. Then it plainly follows by induction that $E_{n}(f+g)=E_{n} f$ $+E_{n} g$, and $\left(E_{n} f\right)\left(x_{1}, \ldots, x_{n}\right) \geq\left(E_{n} g\right)\left(x_{1}, \ldots, x_{n}\right) \quad$ (resp. $\left.\left(E_{n} f\right)\left(x_{1}, \ldots, x_{n}\right)>\left(E_{n} f\right)\left(x_{1}, \ldots, x_{n}\right)\right)$ for ail $\left(x_{1}, \ldots, x_{n}\right) \in R^{n}$ if $f\left(u_{1}, \ldots, u_{n}\right) \geqq g\left(u_{1}, \ldots, u_{n}\right)$ (resp. $\left.f\left(u_{1}, \ldots, u_{n}\right)>g\left(u_{1}, \ldots, u_{n}\right)\right)$ for all $\left(u_{1}, \ldots, u_{n}\right) \in D$.

Next suppose that $f\left(u_{1}, \ldots, u_{n}\right) \geq f\left(v_{1}, \ldots, v_{n}\right)$ (resp. $f\left(u_{1}, \ldots, u_{n}\right)$ $\left.>f\left(v_{1}, \ldots, v_{n}\right)\right)$ for any two distinct $\left(u_{1}, \ldots, u_{n}\right) \in D,\left(v_{1}, \ldots, v_{n}\right) \in D$ such that $Y_{i} \geqslant u_{i} \geqslant v_{i} \geqslant x_{i}$ for all $i=1, \ldots, n$. They by induction $\left(E_{n-1} \operatorname{Tf}\left(u_{1}\right)\right)\left(y_{2}, \ldots, y_{n}\right) \quad \geq \quad\left(E_{n-1} \operatorname{Tf}\right)\left(u_{1}\right)\left(x_{2}, \ldots, x_{n}\right) \quad$ (resp. 


$$
\begin{gathered}
\cdots \\
\because \cdots, \cdots \\
\therefore, \cdots \\
\because \ldots r ?
\end{gathered}
$$


$\left.\left(E_{n-1} \operatorname{Tf}\left(u_{1}\right)\right)\left(y_{2}, \ldots, y_{n}\right)>\left(E_{n-1} \operatorname{Tf}\left(u_{1}\right)\right)\left(x_{2}, \ldots, x_{n}\right)\right)$ for each $u_{1} \in\left\{u\{1), \ldots, u_{m_{1}}^{(1)}\right\}$ and any two distinct $\left(x_{2}, \ldots, x_{n}\right) \varepsilon R^{n-1}$, $\left(y_{2}, \ldots, y_{n}\right) \in R^{n-1}$ such that $Y_{1} \geq u_{1} \geq x_{1}$, and $Y_{i} \geq y_{i} \geq x_{i} \geq x_{i}$ for all $i=2, \ldots, n$. It follows at once from the definition of $E_{1}$ and from (*) that $\left(E_{n} f\right)\left(x_{1}, y_{2}, \ldots, y_{n}\right) \geqslant\left(E_{n} f\right)\left(x_{1}, x_{2}, \ldots, x_{n}\right)$ (resp. $\left.\left(E_{n} f\right)\left(x_{1}, y_{2}, \ldots, y_{n}\right)>\left(E_{n} f\right)\left(x_{1}, x_{2}, \ldots, x_{n}\right)\right)$ for any two distinct $\left(x_{2}, \ldots, x_{y}\right) \in R^{n-1}, \quad\left(y_{2}, \ldots, y_{n}\right) \in R^{n-1}$ and any $x_{1}$ such that $Y_{1} \geq x_{1} \geq$ $X_{1}$ and $Y_{i} \geq y_{i} \geq x_{i} \geq X_{i}$ for $i=2, \ldots, n$. A similar argument shows that $\left(E_{n} f\right)\left(y_{1}, \ldots, y_{n}\right) \geq\left(E_{n} f\right)\left(x_{1}, \ldots, x_{n}\right)$ whenever $Y_{i} \geq y_{i} \geq x_{i} \geq x_{i}$ for all $i=1, \ldots, n$, and that $\left(E_{n} f\right)\left(y_{1}, \ldots, y_{n}\right)>\left(E_{n} f\right)\left(x_{1}, \ldots, x_{n}\right)$ whenever $Y_{i} \geq y_{i} \geq x_{i} \geq x_{i}$ for all $i=1, \ldots, n$ and $\left(y_{1}, \ldots, y_{n}\right),\left(x_{1}, \ldots, x_{n}\right)$ are distinct, provided that $f\left(u_{1}, \ldots, u_{n}\right)>f\left(v_{1}, \ldots, v_{n}\right)$ for any two distinct $\left(u_{1}, \ldots, u_{n}\right) \in D,\left(v_{1}, \ldots, v_{n}\right) \in D$ such that $Y_{i} \geq u_{i} \geq v_{i} \geq X_{i}$ for all $i=1, \ldots, n$. This completes the proof of Lemma 4.2, and with it the decidability of the theory RMMCE.

Q.E.D.

Summarizing we have

Theorem 4.3. The theory RMMCF is decidable.

Remark. RMMCE remains decidable even in the presence of slightly more general kind of up, strict_up predicates. More precisely consider a predicate monotone $\left(f ; a_{1}: x_{1}, y_{1} ; \ldots ; a_{n}: x_{n}, y_{n}\right)$ involving a function variable $f$, real variables $x_{1}, \ldots, x_{n}, y_{1}, \ldots, y_{n}$, and constants $a_{1}, \ldots, a_{n}$ which can take the values 'up', 'strict_up', 'down', 'strict_down'. By definition monotone $\left(f ; a_{1}: x_{1}, y_{1} ; \ldots ; a_{n}: x_{n}, y_{n}\right)$ is satisfied if for every $i \varepsilon\{1, \ldots, n\}$ and every $\xi, n \in\left[x_{1}, y_{1}\right] \times \ldots \times$ $\left[x_{n}, y_{n}\right]$ such that $\xi_{i}<n_{i}$ and $\xi_{j}=n_{j}$ if $j \neq i$, the condition 



$$
a_{i}=\left\{\begin{array}{l}
u p \\
\text { strict_up } \\
\text { down } \\
\text { strict_down }
\end{array}\right\} \text { implies }(M f)(\xi)\left\{\begin{array}{l}
\leq \\
< \\
\geq \\
>
\end{array}\right\}(M f)(n)
$$

Much the same construction as above allows us to prove that the theory RMMCE extended to allow this more general predicate 'monotone' remains decidable. 


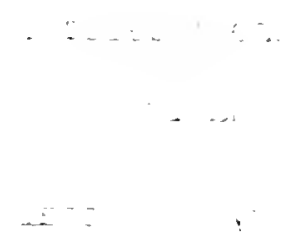

$\therefore: \because$ 
5. Totally and partially ordered sets with monotone functions.

In this section we take up a more abstract problem suggested by the preceding considerations. More specifically, we consider two elementary theories of partially and totally ordered sets, beginning with the 'theory of totally ordered sets with monotone functions'. This theory allows individual variables $x, y, \ldots$ which take elements of a totally ordered set $S$ as values, and also variables denoting (monotone) functions $f_{1}, f_{2}, \ldots$ which are modeled by functions defined on $\mathrm{S}$ and assuming values in S. More formally

Definition 5.1. Terms of the 'theory of totally ordered sets with monotone functions' (TOSME) are:

(i) individual variables;

(ii) expressions of the form $f(t)$, where $f$ is a function variable and $t$ is a term.

Definition 5.2. The atomic formulas of TOSMF are obtained as follows: (i) if $t_{1}$ and $t_{2}$ are terms, then $t_{1}=t_{2}, t_{1}<t_{2}$ are atomic formulas;

(ii) if $f$ is a function variable, then $u p(f)$, strict_up(f), $\operatorname{down}(f)$, strict_down(f) are atomic formulas.

The theory TOSMF is then the Boolean closure of the set of atomic formulas defined above.

Let $\phi$ be a formula of the theory TOSMF.

Definition 5.3. An interpretation is with domain $D$ is said to satisfy $\phi$ if:

(i) $D$ is a totally ordered set; 


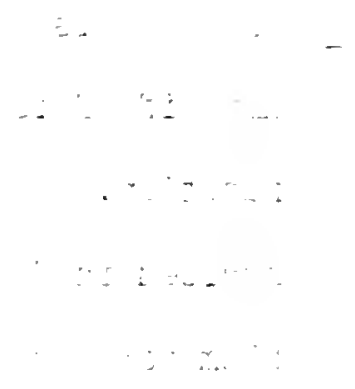


(ii) for every individual variable $x$ in $\phi, M x \in D$;

(iii) for every function variable $f$ occurring in $f$, Mf is a function from $D$ to $D$;

(iv) the formula M $\phi$ obtained fram $\phi$ by substituting every individual variable $\mathrm{x}$ by $\mathrm{Mx}$ and function variable $\mathrm{f}$ by $\mathrm{Mf}$ is true. Here it should be understood that

$\left(i v_{1}\right) u p(f)$ (resp. strict_up(f)) is true in the interpretation $M$ if and only if $M f$ is a nondecreasing (resp. strictly increasing) function.

$\left(\mathrm{iv}_{2}\right)$ down(f) (resp. strict_down(f)) is true in the interpretation $M$ if and only if $M f$ is a nonincreasing (resp. strictly decreasing) function.

Using a normalization process like that described in Section 2, it is easy to show that the satisfiability problem for the theory TOSMF can be reduced to the satisfiability problem for sets of conjunctions of literals each of which has one of the following forms:

$(<)$ $x<y$

$(=)$ $\mathrm{x}=\mathrm{y}$

$$
\mathrm{x}=\mathrm{f}(\mathrm{y})
$$

$(u-d)$

$$
u p(f), \operatorname{down}(f)
$$

(strict) strict_up(f), strict_down(f)

$(\mid u-d) \quad-|u p(f),-| \operatorname{down}(f)$

(|strict) |strict_up(f), |strict_down(f).

Let $\phi$ be a conjunction of literals of the types $(<,=, f, u-d$, strict, lu-d, |strict). As usual we can easily eliminate clauses in $\phi$ of types ( $|u-d,-|$ strict), and thus can assume that no clause of the type $(-|u-d,-|$ strict $)$ occurs in $\phi$. We will show that $\phi$ is satisfiable 


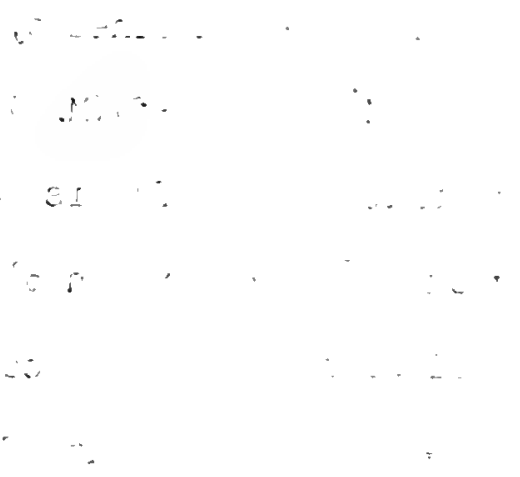


(according to the satisfiability definition given above) if and only if $\phi$ has a real model, that is a model whose domain is the real axis $\mathbb{R}$ with the standard order in it. Plainly any real model is a model of $\phi$. Conversely, assume that $M$ is a model of $\phi$. Then a real model of $\phi$ can be built as follows. Let $T=\{M x \mid x$ occurs in $\phi\} \cup\{(M f)(M x) \mid f, x$ occur in $\phi\}$. Clearly $T$ is a totally ordered finite subset of the domain $D$ of M. Hence $T$ can be embedded in $\mathbb{R}$. Let $I: T \rightarrow \mathbb{R}$ be such an embedding. For any individual variable $x$ occurring in $\phi$, put

$$
M^{*} X=I(M X)
$$

Moreover with any function variable $f$ occurring in $\phi$, we associate a function $E:\left\{M^{*} x \mid x\right.$ occur in $\left.\dot{\varphi}\right\} \rightarrow \mathbb{R}$ defined by

$$
F\left(M^{*} x\right)=I((M P)(M x)) \text {. }
$$

We begin by showing that if $u p(f)$ is a conjunct of $\phi$, then $F$ is monotone nondecreasing on $\left\{M^{*} x \mid x\right.$ occurs in $\left.\phi\right\}$. Indeed, let $M^{*} x_{1} \leq M^{*} x_{2}$ for sone pair of variables $x_{1}, x_{2}$ occurring in $\phi$. Then $M x_{1}=$ $I^{-1}\left(M^{*} x_{1}\right) \leq I^{-1}\left(M^{*} x_{2}\right)=M x_{2}$, so that $F\left(M^{*} x_{1}\right)=I\left((M f)\left(M x_{1}\right)\right) \leq$ $I\left((M f)\left(M x_{2}\right)\right)=F\left(M^{*} x_{2}\right)$. Similarly it follows that if strict_up(f), down(f), or strict_down(f) appear in $\phi$, then $F$ is monotone increasing, nonincreasing or decreasing (respectively). Therefore $F$ can be extended to an everywhere continuous function $\bar{F}$ in such a way that if $u p(f), \quad$ strict_up(f), down(f), strict_down(f) occur in $\phi$, then $\bar{E}$ is respectively monotone nondecreasing, increasing, nonincreasing or decreasing. Finally we put $M^{*} f=\bar{F}$, for any function variable $f$ occurring in $\phi$. It then is an easy matter to verify that $M^{*}$ is a real model of $\varphi$. Hence the decidability of the theory TOSilf follows from Theorem 3.4 . 





Theorem 5.4. The theory TOSMF is decidable.

Next we consider the somewhat different theory of partially ordered sets with (monotone) functions (POSMF) and show that its satisfiability problem is decidable also. More precisely we will refer to those partially ordered sets in which any two elements have both a Iower and an upper bound. The syntax of the theory POSMF is the same as the one of the theory TOSMF, with the only exception that no 'strict' predicate is allowed in this case. Moreover we define satisfiability as follows.

Definition 5.5. Let $\phi$ be a formula of the theory POSMF. An interpretation $M$ with domain $D$ is said to satisfy $\phi$ if:

(i) $D$ is a partially ordered set such that any two elements in $D$ have both a lower and an upper bound, and in which the relationship $\mathrm{x}$ $\leqq y \leqq x$ implies that $x=y$.

(ii) for every individual variable $x$ in $\phi, M x \in D$;

( $i$ ii) for every function variable $f$ occurring in $\phi$, $M f$ is a function from $D$ to $D$;

(iv) the formula $M \phi$ obtained from $\phi$ by replacing $x$ by $M x$ and $f$ by $M f$, wherever an individual variable $x$ or a function variable $f$ occurs in $\phi$, is true, and where we agree that

$\operatorname{lu}(f)$ (resp. down $(f)$ ) is true in the interpretation $M$ if and only if $\mathrm{Mf}$ is a monotone nondecreasing (resp. nonincreasing) function;

Normalizing as before the satisfiability problem for the theory POSMF is easily reduced to the satisfiability problem for sets of literals of the form 


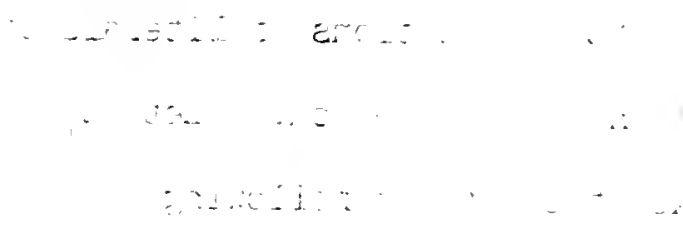




$\begin{array}{ll}(\underline{)}) & x \leq y \\ (\underline{x}) & x \underline{x y} \quad(i \cdot e \cdot-\mid(x \leq y)) \\ (\Rightarrow) & x=y \\ (f) & x \neq y \\ (f) & x=f(y) \\ (u-d) & u p(f), \operatorname{down}(f) \\ (-\mid u-d) & |u p(f),-| \operatorname{down}(f) .\end{array}$

Moreover, since clauses $(\lceil\mid u-d)$ can be eliminated quite easily (cf. the simplification step 1 of the preceding section) we can further restrict our attention to conjunctions of literals of the type $(\leq)-$ (u-d). Hence let $\phi$ be such a conjunction. Let $\phi_{1}$ be the formula obtained from $\phi$ after execution of the following

\section{Simplification Step.}

(a) For every pair of clauses of $\phi$ of the form $x=f(y), x^{\prime}=f\left(y^{\prime}\right)$ add the formula $y=y^{\prime} \rightarrow x=x^{\prime}$

(b) For every triple of clauses of $\phi$ of the form up(f) (resp. down $(f)), \quad x=f(y), \quad x^{\prime}=f\left(y^{\prime}\right)$ add the formula $y \leq y^{\prime} \rightarrow x \leq x^{\prime}$ (resp. $\left.y \leq y^{\prime} \rightarrow x^{\prime} \leq x\right)$.

(c) For every quadruple of clauses of $\phi$ of the form $u p(f)$, down(f), $x$ $=f(y), x^{\prime}=f\left(y^{\prime}\right)$ add the formula $x=x^{\prime}$.

(d) Drop all clauses of $\phi$ involving function variables.

Clearly any model of $\phi$ is also a model of $\phi_{1}$, since formulas added by the simplification substeps (a), (b) and (c) are consequences of $\phi$. For example, by (i) of Definition 5.5 it follows that any function which is both increasing and decreasing must be a constant function, 


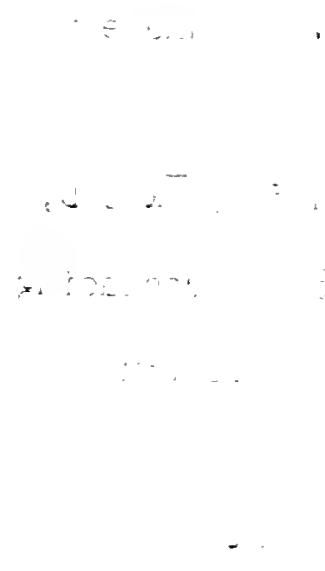


which shows the correctness of substep (c). Let $\bar{\phi}_{1}$ be the formula obtained from $\phi_{1}$ by replacing every occurrence of the relation symbols $\leqq, \&$ with $\subseteq$ and $\underline{q}$ respectively, where $\subseteq$ is the ordinary set inclusion symbol.

We will now show that if $\phi_{1}$ has a partially ordered model, then $\bar{\phi}_{1}$ has a set model defined as follows:

Definition 5.6. An interpretation $\bar{M}$ with domain $D$ is a set model of $\bar{\phi}_{1}$ if

(i) there is a set 3 such that $D=$ pow $(S)$ (where pow(S) is the set of all subsets of $S$ ) :

(ii) for every individual variable $x$ in $\Phi_{1}, \bar{M} x \in D$;

(iii) the formula $\bar{M} \Phi_{1}$ obtained from $\bar{\phi}_{1}$ by replacing $\mathrm{x}$ by $\bar{M} \mathrm{x}$ for each individual variable $x$ occuring in $\Phi_{1}$ is true.

To prove our assertion, let $M$ be a partially ordered model of $\phi_{1}$ having domain $S$. We will build a set model $\bar{M}$ of $\Phi_{1}$ with domain $D=$ pow $(S)$. Let $h: S \rightarrow \operatorname{pow}(S)$ be the mapping defined by $h(s)=\{t \varepsilon S \mid t \leq s\}$ (where $\leq$ is the order relation in $S$ ). For every individual variable $x$ in $\bar{\phi}_{1}$, we put

$$
\bar{M}_{X}=h(M X)
$$

Let $\mathrm{x} \subseteq \mathrm{y}$ be a clause of $\bar{\phi}_{1}$. Then $\mathrm{x} \leq \mathrm{y}$ is a clause in $\phi_{1}$, and since $M x \leq M y$, it follows by the transitivity of $\leq$ and the definition of $h$ : $S$ $\rightarrow \operatorname{pow}(S)$ that $h(M x) \subseteq h(M y)$, i.e. $\bar{M} x \subseteq \bar{M} y$. Therefore $\bar{M}$ satisfies all literals in $\bar{\phi}_{1}$ of the type $(\subseteq)$. Similarly it can be shown that $\bar{M}$ also satisfies all literals in $\bar{\varphi}_{1}$ of the remaining types including the 
xax:




implicative clauses introduced in step (a) and (b), which proves that $\bar{M}$ is a set model of $\Phi_{1}$.

Moreover any set model $\bar{M}$ of $\Phi_{1}$ can be used to define a partially ordered model $M$ of $\phi$. Indeed, the relation $\subseteq$ is a partial order of the domain $D=$ pow $(S)$ of $\bar{M}$, so that all literals in $\phi$ of the form ( $\leq$, $\mathbf{x},=, \not)$ are correctly modeled by $\bar{M}$ if $\leq$ is interpreted as set inclusion, and clearly $\mathrm{x} \cap \mathrm{y}$ and $\mathrm{x} \cup \mathrm{y}$ are respectively $\mathrm{a}$ lower and an upper bound of $x, y \in D$. Hence all we need to do in order to get a model of $\phi$ is to extend $\bar{M}$ to function variables in such a way that clauses of types $(f, u-d)$ are correctly modeled too.

For every function variable $f$ occurring in $\phi$ for which up( $f$ ) occurs in $\phi$ while down( $f)$ does not occur in $\phi$, and for any $s \varepsilon S$, put

$$
(\bar{M} f)(s)=\underset{x \varepsilon A_{S}}{U} \overline{M x}
$$

where $A_{S}=\{x j x=f(y)$ occurs in $\phi$ and $\bar{M} y \subseteq s\}$.

For every function variable $f$ occurring in $\phi$ for which down(f) occurs in $\phi$ while up(f) does not occur in $\phi$, and for any $s \varepsilon S$, put

$$
(\bar{M} f)(s)=\bigcap_{X \in A_{S}} \bar{M} X
$$

If both $u p(f)$ and down(f) occur in $\phi$, put

$$
(\bar{M} f)(s)=\bar{V} x
$$

for some $x=f(y)$ occurring in $\phi$. Observe that (5.3) does not depend 


$$
\therefore=+0 \cdot \frac{2}{2}=
$$


on the clause $x=f(y)$ chosen since by the Simplification substep (c) $\overrightarrow{M x}=\bar{M} x^{\prime}$ if both $x=f(y)$ and $x^{\prime}=f(y)$ occur in $\phi$. If neither $u p(f)$ nor down(f) occurs in $\phi$, define $\bar{M} f$ to be any extension to the whole of $D$ of the set-to-set mapping $F$, defined by

$$
F(\bar{M} y)=\bar{M} x \text { if } x=f(y) \text { occurs in } \phi .
$$

(Note that by the Simplification Substep (a), this definition is legal.) Plainly if neither up(f) nor down(f) occurs in $\phi$, all clauses of type (f) are satisfied by this definition.

We must show that if $u p(f)$ is in $\phi$ but $\operatorname{down}(f)$ does not occur in $\phi$, then $\bar{M} f$ is monotone nondecreasing, with respect to the relation $\subseteq$. To this end, let $s, t \in S$, such that $s \subseteq t$. Clearly $A_{S} \subseteq A_{t}$, and therefore by $(5.1)$

$$
(\bar{M} f)(s)=\underset{X \in A}{\cup} \overline{M X} \subseteq \underset{X \in A_{t}}{U} \bar{M} X=(\bar{M} f)(t),
$$

which shows that $\bar{M} f$ is monotone nondecreasing. Next, suppose that $u p(f)$ occurs in $\phi$ and that $x^{\prime}=f\left(y^{\prime}\right)$ is a literal of $\phi$. To be sure that all type (f) clauses are satisfied in this case also we must prove that $(\bar{M} f)\left(\bar{M}^{\prime}\right)^{\prime}=\bar{M}^{\prime}$. Let $x=f(y)$ be any literal in $\phi$ such that $\bar{M} y \subseteq \bar{M}_{y^{\prime}}$. Since $\bar{\phi}_{1}$ contains the conjuncts $y \subseteq y^{\prime} \rightarrow x \subseteq x^{\prime}$ and $y=y^{\prime} \rightarrow$ $x=x^{\prime}$ (which are introduced into $\phi_{1}$ by the Simplification Substeps (b)

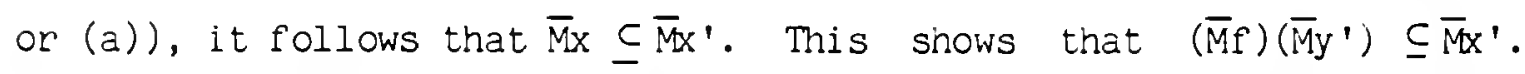
However, $(\bar{M} f)\left(\bar{M} y^{\prime}\right) \supseteq \bar{M}_{x^{\prime}}$, since $x^{\prime}=f\left(y^{\prime}\right)$ occurs in $\phi$ and $\bar{M} y^{\prime} \subseteq \bar{M} y^{\prime}$. Thus $(\bar{M} f)\left(\bar{M} y^{\prime}\right)=M^{\prime}$.

A very similar argument shows that if down(f) occurs in $\phi$ but up(f) does not, then (5.2) correctly models both the statement down(f) and all clauses $x=f(y)$ involving the function variable $f$. The case 
$\therefore$ 
in which both $u p(f)$ and down(f) occur in $\phi$ is even easier. Taken all together, this shows that $\bar{M}$ models the set $\phi$ of statements correctly.

It follows that $\phi$ is satisfiable if and only if $\Phi_{1}$ has a set model. Since $\Phi_{1}$ is a trivial subset of the decidable theory MLS (see [FOS 80], cf. also [Qui50]), we can conclude that

Theorem 5.7. The theory POSMF is decidable. 







\section{References}

[Col75] Collins, G., "Quantifier Elimination for Real Closed Fields by Cylindrical Algebraic Decomposition," in "Second GI Conference on Automata Theory and Formal Languages," Lect. Notes in Comp. Sci., Vol. 33, Springer-Verlag, Berl in, 1975.

[Dan63] Dantzig, G. B., "Linear Programming and Extensions," Princeton University Press, Princeton, 1963.

[FOS80] Ferro, A., Omodeo, E., Schwartz, J. T., "Decision Procedures for Elementary Sublanguages of Set Theory. I. Multi-Level Syllogistic and Some Extensions," Comm. Pure Appl. Math., Vol. XXXIII, 1980.

[Qui50] Quine, W. V., "Methods of Logic," Henry Holt, New York, 10-).

[Tar51] Tarski, A., "A Decision Method for Elementary Algebra and Geometry," 2nd ed. rev., Univ. of California Press, Berkeley, 1951. 
NYU COMPSCI TR-217 C.I

Cantone, D

Decision algorithms for

some fragments of analysis and related areas

NYU COMPSCI TR-217 C.I
Cantone, D

- Decision algorithms for some fragments of analysis

* and related areas

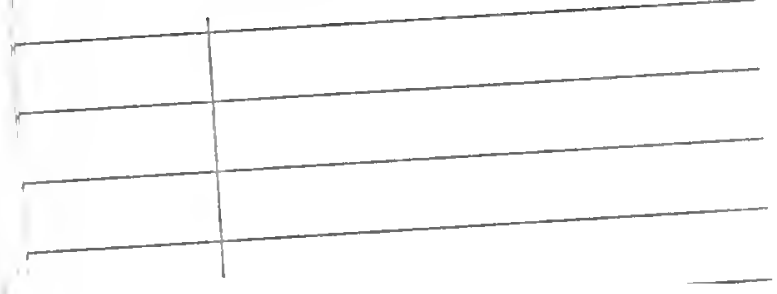

\section{LIBRARY}

N.Y.U. Courant Institute of

\section{Mathematical Sciences}

This book may be kept

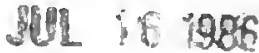

\section{FOURTEEN DAYS}

A foe will be charged for each day the book is kept overtine.

\begin{tabular}{l|l|l|l}
\hline & & & \\
\hline & & & \\
\hline & & & \\
\hline & & & \\
\hline & & & \\
\hline & & & \\
\hline & & & \\
\hline & & & \\
\hline & & & \\
\hline & & & \\
\hline & & & \\
\hline
\end{tabular}




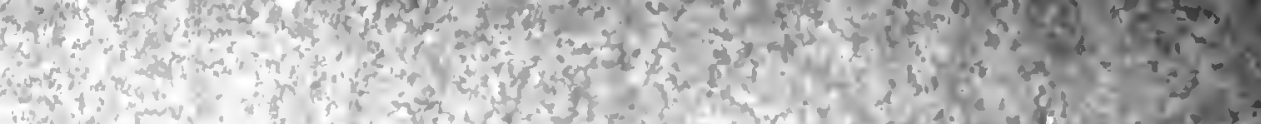

